\title{
Efecto de la diversidad funcional sobre la tasa de evapotranspira- ción: un análisis anual de tres bosques templados chilenos
}

\section{Effects of functional diversity on evapotranspiration rate: an annual analysis of three Chilean temperate forests}

\author{
Jocelyn Esquivel ${ }^{1,2 *}$, Aníbal Pauchard ${ }^{1,2} \&$ Alfredo Saldaña $^{3}$ \\ 1Departamento de Manejo de Bosques y Medio Ambiente, Facultad de Ciencias Forestales, Universidad de Concepción, Concepción, Chile. \\ ${ }^{2}$ Instituto de Ecología y Biodiversidad (IEB), Chile. \\ ${ }^{3}$ Departamento de Botánica, Facultad de Ciencias Naturales y Oceanográfıcas, Universidad de Concepción, Concepción, Chile. \\ *Email: jesquivel@udec.cl
}

\section{RESUMEN}

Existe un vínculo entre la diversidad de ecosistemas forestales y el proceso ecosistémico evapotranspiración (ET). Sin embargo, hay un escaso conocimiento de este vínculo en bosques templados del sur de Sudamérica. Dada la influencia de la ET en servicios ecosistémicos relevantes, como la regulación hidrológica o climática, es importante comprender el efecto del cambio en la composición de las especies sobre este proceso. El objetivo de esta investigación fue determinar la relación entre diversidad funcional y la variación estacional de ET usando como modelo de estudio tres ecosistemas forestales nativos que difieren en estructura, composición de especies dominantes y proporción de estrategias fenológicas. El patrón general muestra que existe una relación negativa significativa entre la media ponderada comunitaria del atributo área foliar específica; CWMSLA y el proceso ET. Encontramos cambios estacionales en ET en los tres ecosistemas, la que fue mayor en primavera-verano. Existe una relación positiva entre el porcentaje de especies siempreverdes en el dosel y ET total anual de cada ecosistema. Esto explicaría la relación negativa entre CWM-SLA y ET, ya que el ecosistema que presenta mayor proporción de especies siempreverdes corresponde al de menor CWM-SLA. Los resultados de este estudio contribuyen a dar relevancia a la diversidad funcional de ecosistemas forestales nativos sobre un proceso ecosistémico crucial para la provisión de diversos servicios ecosistémicos. Estudios posteriores podrían evaluar el efecto de la pérdida de diversidad funcional sobre procesos ecosistémicos con el fin de relevar la necesidad de conservar y/o restaurar el bosque nativo desde esta perspectiva.

Palabras clave: área foliar específica, bosque templado, diversidad funcional, evapotranspiración, proporción caducifolias/siempreverdes.

\section{ABSTRACT}

There is a link between the diversity of forest ecosystems and the evapotranspiration (ET) ecosystem process. However, there is little knowledge of this link in temperate forests of southern South America. Given the influence of ET in relevant ecosystem services, such as hydrological or climatic regulation, it is important to understand the effect of the change in species composition on this process. The objective of this research was to determine the relationship between functional diversity and the seasonal variation of ET using three native forest ecosystems that differ in structure, composition of dominant species and proportion of phenological strategies as a study model. The general pattern shows that there is a significant negative relationship between the community weighted average of the specific leaf area attribute; CWM-SLA and the ET process. We found seasonal changes in ET in the three ecosystems, which was greater in spring-summer. There is a positive 
relationship between the percentage of evergreen species in the canopy and total annual ET of each ecosystem. This would explain the negative relationship between CWM-SLA and ET, since the ecosystem with the highest proportion of evergreen species corresponds to the one with the lowest CWM-SLA. The results of this study contribute to giving relevance to the functional diversity of native forest ecosystems over a crucial ecosystem process for the provision of various ecosystem services. Subsequent studies could assess the effect of the loss of functional diversity on ecosystem processes in order to relieve the need to conserve and/or restore the native forest from this perspective.

Keywords: deciduous/evergreen ratio, evapotranspiration, functional diversity, specific leaf area, temperate forest.

\section{INTRODUCCIÓN}

La diversidad biológica, definida como el número, abundancia, composición, distribución espacial e interacciones de genotipos, poblaciones, especies, tipos y rasgos funcionales, y unidades del paisaje en un determinado sistema (Díaz et al. 2006), determina fuertemente el funcionamiento ecosistémico y los procesos asociados (Díaz \& Cabido 2001). Dentro de los numerosos procesos ecosistémicos, la evapotranspiración (ET), que describe el transporte de agua desde la superficie terrestre a la atmósfera, es un proceso relevante para importantes servicios ecosistémicos de regulación, tales como: balance hídrico y regulación climática (Hein et al. 2006; Ward \& Trimble 2003). Es además un proceso importante en el transporte de nutrientes en las plantas y la dinámica del ecosistema (Kelliher et al. 1995), ya que también está estrechamente vinculado al proceso de captura de carbono y productividad primaria (Mo et al. 2018). El control fisiológico de la ET responde tanto a factores propios del equilibrio interno de la vegetación (Pieruschka et al. 2010), como a factores externos, tales como: radiación neta, velocidad del viento, temperatura, agua disponible en el suelo y déficit de vapor de agua en el aire (Schulze et al. 2005). La influencia relativa de estos factores sobre el control estomático, y por ende sobre el proceso de evapotranspiración, depende principalmente de las propiedades estructurales de la vegetación presente, lo cual a su vez depende de la identidad de las especies que forman esta, ya que las plantas regulan la pérdida de agua principalmente a través de su forma (Schulze et al. 2005). Por lo tanto, la influencia del dosel de la vegetación sobre la evapotranspiración es también función de la diversidad de plantas que componen el ecosistema, de su morfología y de su configuración espacial (Baudena et al. 2013). Es por esto que las características de las plantas tienen el potencial en explicar la variación de procesos de intercambio gaseoso entre el ecosistema y la atmósfera (Reichstein et al. 2014), siendo los atributos foliares los que tienen mayor influencia sobre la evapotranspiración en particular (Martín-López et al. 2007; Schulze et al. 2005)

La relación entre procesos ecosistémicos y biodiversidad ha sido estudiada principalmente en función del atributo composición, donde el principal indicador utilizado es la riqueza de especies y sus abundancias relativas (Tilman \& Lehman 2002). Sin embargo, la diversidad funcional, definida como el valor, rango, distribución y abundancia relativa de los rasgos funcionales de las especies que componen un determinado ensamble, comunidad o ecosistema (Díaz et al. 2007; Díaz \& Cabido 2001; Tilman 2001), parece ser un mejor predictor de la función ecosistémica que la riqueza de especies (Cadotte et al. 2011; Hillebrand \& Matthiessen 2009; Petchey \& Gaston 2006). Un atributo funcional es cualquier expresión fenotípica, ya sea morfológica, fisiológica o fenológica, que impacta indirectamente a la adecuación biológica del individuo, a través de su efecto sobre el crecimiento, la supervivencia y la reproducción de este (Violle et al. 2007). Se postula que el incremento en diversidad funcional tendría un efecto positivo sobre la tasa de los procesos ecosistémicos (e.g. productividad primaria, balance hídrico, ciclaje de nutrientes), debido a un mejor uso complementario de los recursos (Díaz \& Cabido 2001; Hooper \& Vitousek 1997; Tilman et al. 1997). En este contexto, una alta divergencia funcional indicaría un alto grado de diferenciación de nichos y baja competencia, por lo que comunidades con un alto valor para este parámetro pueden optimizar el funcionamiento de algún proceso ecosistémico dado producto de las diferencias interespecíficas en la captura y utilización de recursos (Mason et al. 2005). Por otra parte, dentro de un ecosistema podemos encontrar comunidades cuyas especies en conjunto presenten, para un determinado rasgo funcional, un valor promedio que se asocie positivamente con una medida de funcionamiento ecosistémico (Garnier et al. 2004; Díaz \& Cabido 2001). De tal manera, la pérdida o adición de especies 
con ciertos rasgos funcionales puede tener un gran impacto en un proceso ecosistémico en particular, mientras la pérdida de otras especies tendría menor e inclusive nulo impacto, ya que distintos procesos son susceptibles de ser afectados por diferentes especies y/o grupos funcionales (Díaz \& Cabido 2001; Tilman et al. 1997), principalmente si los rasgos de una o pocas especies son los que dominan la comunidad (Hooper \& Vitousek 1997). Grime (1998) estableció que la productividad de un ecosistema, y por ende los procesos ecosistémicos asociados (e.g. evapotranspiración; Lambers et al. 2008), dependen en mayor medida de los rasgos funcionales de las especies dominantes y de la contribución de dichas especies a la biomasa total de la comunidad, las que tendrían un mayor efecto sobre los procesos ecosistémicos (Mokany et al. 2008).

Aun cuando se ha planteado la importancia de la diversidad funcional en el funcionamiento ecosistémico y en la provisión de servicios asociados, la utilización de este componente de la diversidad en la evaluación de dichos servicios es incipiente, y por ende dicho componente ha sido escasamente considerado en la planificación, manejo o toma de decisiones en recursos naturales (Martín-López et al. 2007). En este sentido es importante destacar que para el caso particular de los ecosistemas de bosque nativo en Chile, algunas investigaciones, como la de Lara et al. (2009), sugieren la importancia de este proceso ecosistémico en la provisión de servicios ecosistémicos de regulación y calidad de agua. Sin embargo, en estudios de este tipo normalmente no se mide o estima la evapotranspiración como proceso relevante en la función de la vegetación, o bien el bosque nativo es clasificado con base en su estructura, bosques primarios o secundarios, pero sin considerar la identidad y rasgos funcionales de las especies que lo componen, ni cómo esta característica influye en los procesos ecosistémicos. Incluso cuando se estiman algunos servicios ecosistémicos como provisión de agua a nivel de cuenca, en la parametrización de los modelos que se utilizan para dicha estimación se considera que los valores de variables como la evapotranspiración son aproximadamente similares para cualquier tipo de cobertura de bosque nativo (Stehr et al. 2010). Además, en ecosistemas con presencia de especies caducifolias, el efecto que tendrá la pérdida total o parcial de la cobertura del dosel en el periodo de senescencia sobre la intercepción de la precipitación y los procesos de evapotranspiración e infiltración, son importantes de considerar al evaluar el efecto del bosque nativo sobre el ciclo hidrológico. Se ha demostrado que la escorrentía superficial total anual difiere de la escorrentía estacional dependiendo del tipo de vegetación presente. En este sentido, en bosques mixtos (i.e. caducifolias siempreverdes) considerar funcionalmente el rol de las especies caducifolias en relación con el de las siempreverdes resulta relevante, ya que de la representatividad de cada uno de estos grupos en la comunidad, dependerá la regulación del proceso de evapotranspiración. Lara et al. (2009) reportaron variabilidad estacional en la escorrentía superficial de seis cuencas andinas del centro-sur de Chile, donde la escorrentía de verano es mayor en bosque nativo que en plantaciones de Pinus radiata D. Don y Eucalyptus spp., lo que denota la importancia de considerar la estacionalidad del proceso en zonas donde los bosques nativos presentan fenología mixta (caducifolio-siempreverde).

Dada la influencia de la diversidad de la vegetación en procesos ecosistémicos como la evapotranspiración, y al escaso conocimiento que se tiene de este vínculo para el bosque templado del sur de Sudamérica, es necesario mejorar el entendimiento de cómo la diversidad funcional afecta dicha función ecosistémica. Determinar que la diversidad funcional y la evapotranspiración difieren entre comunidades de bosque nativo, permitirá mejorar las predicciones del efecto del cambio en la composición de especies (e.g. degradación de ecosistemas, pérdida de especies, invasiones biológicas, etc.) sobre la función ecosistémica y por ende en la provisión de servicios. El objetivo de esta investigación es determinar la relación entre diversidad funcional y el proceso evapotranspiración usando como modelo de estudio tres ecosistemas forestales templados, que convergen en el Parque Nacional Tolhuaca y que están sometidos a una misma zona bioclimática (Luebert \& Pliscoff 2006). Se espera que i) la evapotranspiración varíe entre ecosistemas de bosque nativo en función de sus atributos funcionales; ii) que ecosistemas con mayor diversidad funcional de atributos foliares presenten mayor tasa de evapotranspiración.

\section{MÉTODOS}

ÁREA DE ESTUDIO Y SELECCIÓN DE ECOSISTEMAS

El área de estudio se encuentra ubicada en el centro-sur de Chile entre los $38^{\circ} 5^{\prime} ; 71^{\circ} 58^{\prime}$ y $38^{\circ} 12^{\prime}$; $71^{\circ} 45^{\prime}$ (Fig. 1). El área cuenta con remanentes de bosque nativo primarioy secundario asociados al Parque Nacional Tolhuaca. Incluye ecosistemas con diferentes atributos estructurales, composicionales y funcionales, ubicados en la misma región bioclimática, correspondiente a un clima Templado-Hiperoceánico.

Se seleccionaron ecosistemas con distinta estructura y composición. En un primer análisis se utilizó la clasificación supervisada de una imagen Landsat 7 ETM del año 2011, para determinar la estructura del bosque nativo, el que fue clasificado en bosques primarios y secundarios. La imagen Landsat 7 ETM seleccionada fue sometida a corrección geométrica utilizando puntos aleatoriamente seleccionados. 
Luego mediante el software IDRISI se aplicó corrección radiométrica y topográfica respectivamente, para reducir los efectos atmosféricos en la calidad de la imagen (Chavez 1996) y el efecto de las sombras por la topografía local (Hantson \& Chuvieco 2011; Reese \& Olsson 2011; Teillet. et al. 1982). Luego, para llevar a cabo la clasificación supervisada, se utilizó el software ENVI 4.5, el cual se basa en el estadístico de máxima similitud y puntos de entrenamiento (Segura \& Trincado 2003). Se utilizaron 350 puntos de entrenamiento obtenidos mediante campañas de terreno y fotografías aéreas.

Luego, se utilizó la cartografía de la clasificación de los pisos vegetacionales de Chile (Luebert \& Pliscoff 2006), con el fin de seleccionar áreas con una superficie superior a 5 hectáreas. Luego de la selección de áreas se verificó en terreno la composición y estructura para definir los ecosistemas adecuados para el estudio. Se definieron tres repeticiones para cada ecosistema evaluado, los que correspondían a: ecosistema 1 (E1) del piso vegetacional Bosque resinoso templado andino de Araucaria araucana y Nothofagus dombeyi, corresponde a un bosque primario dominado por la especie Araucaria araucana, especie longeva siempreverde, acompañada en el dosel superior por dos especies siempreverdes y una caducifolia. El ecosistema 2 (E2), del piso vegetacional Bosque caducifolio templado andino de Nothofagus Alpina y N. dombeyi, es un bosque primario dominado por Nothofagus dombeyi, es el ecosistema con mayor riqueza de especies en el dosel superior y presenta mayor dominancia de especies siempreverdes. El ecosistema 3 (E3), también, corresponde al piso vegetacional anterior, pero presenta una estructura diferente, ya que es un bosque secundario dominado por $N$. dombeyi y es el único que presenta igual proporción de especies siempreverdes y caducifolias.

\section{DiversidAD FUNCIONAL}

Para determinar la diversidad funcional de las zonas seleccionadas se utilizaron los índices Media Comunitaria Ponderada (CWM) (Garnier et al. 2004) y Divergencia Funcional (FDvg) (Lepš et al. 2006; Mason et al. 2005), los que fueron calculados utilizando el software FDiversity (Casanoves et al. 2011). Ambos índices se basan en la medida de un rasgo funcional. El rasgo evaluado fue área foliar específica (SLA), ya que es un atributo adecuado para analizar la diversidad funcional a nivel comunitario (Poorter \& Garnier 1999; Reich et al. 1997), y corresponde a la relación entre el área foliar que captura luz por unidad de masa seca (Reich et al. 2003). Para obtener el SLA se estableció un muestreo estratificado en cada tipo de ecosistema evaluado.

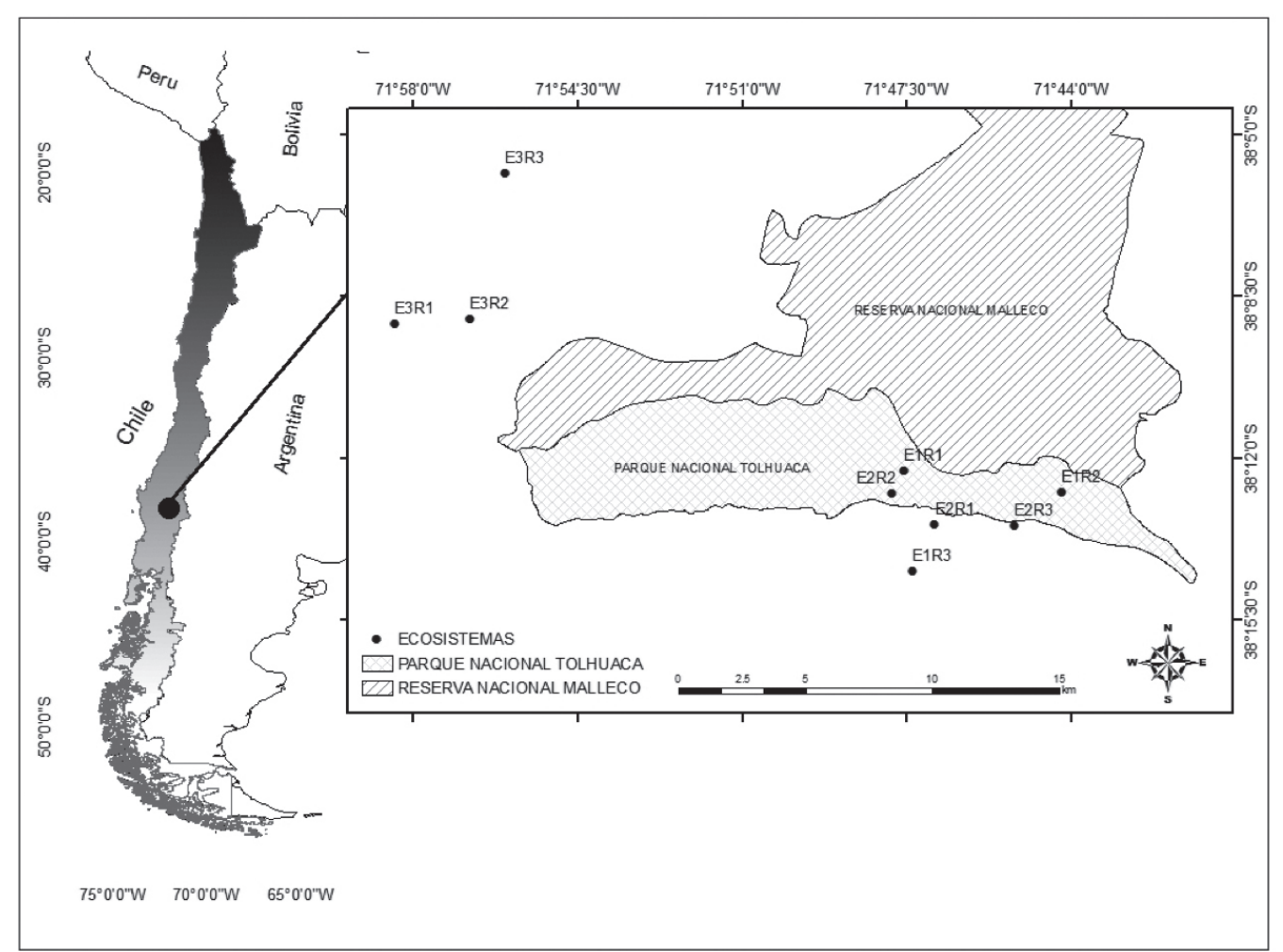

Figura 1. Ubicación geográfica del área de estudio y ecosistemas boscosos. / Geographical location of the study area and forest ecosystems. 


\section{TOMA DE MUESTRAS}

Para determinar SLA se recolectaron 30 hojas de cada una de las especies dominantes por tipo de ecosistema, de acuerdo con el protocolo definido por Pérez-Harguindeguy et al. (2013). Para estimar la abundancia por especie se establecieron tres parcelas de $10 \times 20 \mathrm{~m}$ en cada tipo de ecosistema y sus respectivas repeticiones.

Media Comunitaria Ponderada de un atributo

$$
C W M_{\text {trait }}=\sum_{i=1}^{n} p_{i} \text { x } \text { atributo }_{i}
$$

Donde:

$\mathrm{p} i=$ contribución relativa de la especie i a la biomasa de la parcela.

$\mathrm{n}=$ número de especies.

atributo $=$ valor promedio de un atributo dado en la especie i (SLA).

Considera las abundancias relativas de las especies. CWM refleja el valor de rasgo dominante en la comunidad.

Divergencia funcional

$$
F D_{v g}=\frac{2}{\pi \arctan }\left[5 x \sum_{i=1}^{n}\left[(\ln C i-\overline{\ln x})^{2} x A_{i}\right]\right]
$$

Donde:

FDvg = divergencia funcional para los intervalos de clase de la distribución de frecuencia de un atributo funcional dado.

$\mathrm{C}_{i}=$ valor del atributo para el intervalo de clase iavo.

$\mathrm{A} i=$ abundancia proporcional de la especie $\mathrm{i}$ en el intervalo de clase.

In $\mathrm{x}=$ media ponderada por abundancia del In de los valores del atributo en cada intervalo de clase.

La divergencia funcional es un indicador directo de la diferenciación de nicho entre las especies de cada comunidad, el cual varía entre 0 y 1 , adquiriendo valor 1 cuando la abundancia es mayor hacia uno o ambos márgenes de la distribución del rasgo. Los valores inferiores se obtienen cuando la abundancia se concentra hacia el valor medio de ese rasgo en una comunidad (Butterfield \& Suding 2013; Mason et al. 2005).

\section{EVAPOTRANSPIRACIÓN}

La evapotranspiración fue evaluada mediante información satelital (información ex situ) y un modelo de estimación (información in situ). Para la evaluación ex situ de la evapotranspiración se utilizaron dos fuentes de información satelital: MODIS y Landsat TM (Thematic Mapper), del año 2014, ambos de distinta resolución espacial y temporal. El producto MODIS MOD16 corresponde a mapas de evapotranspiración, generados por el proyecto NASA/EOS, el cual estima la evapotranspiración terrestre mediante un algoritmo cuya base es la ecuación de Penman-Monteith (Monteith 1965). La evapotranspiración estacional se obtuvo mediante la suma de evapotranspiración total mensual, en milímetros, para cada estación correspondiente. Se definió como verano los meses de enero a marzo; otoño, de abril a junio; invierno, de julio a septiembre y primavera, de octubre a diciembre, considerando para esto los cambios fenológicos observados en las especies en el área de estudio.

Las imágenes Landsat TM poseen una resolución espacial de $30 \mathrm{~m}$ y fueron evaluadas mediante el modelo METRIC (Mapping Evapotranspiration at high Resolution using Internalized Calibration). METRIC estima evapotranspiración como el residual del balance de energía, donde el flujo de calor latente es función de la radiación neta, el calor del suelo y el flujo de calor sensible. METRIC utiliza además información meteorológica de estaciones cercanas al área de estudio. El uso de ambas fuentes de información satelital permitió obtener la estacionalidad del proceso evapotranspiración y validar los resultados obtenidos.

Un método de estimación de evapotranspiración indirecto que considera evaluación de variables in situ, corresponde al uso del modelo Hargreaves \& Samani (1985). Este modelo ha demostrado excelentes resultados en la estimación de evapotranspiración, al ser comparado con los resultados de un lisímetro (Jensen et al. 1990), y para aplicarlo se necesita escasa información meteorológica, lo que facilita su uso. Se estimó evapotranspiración de referencia, valor que se multiplica por un coeficiente de cultivo para bosques para obtener evapotranspiración real. El coeficiente de cultivo fue corregido según el índice de área foliar (IAF), definido como la cantidad de área foliar por unidad de área de superficie, según la metodología establecida por Allen et al. (1998). El IAF fue medido en cada tipo de ecosistema mediante fotografías hemisféricas, obteniendo un coeficiente de cultivo para cada tipo de bosque. La evapotranspiración diaria se llevó a evapotranspiración estacional, con el fin de comparar los resultados con los obtenidos en base a información satelital.

Considerando las marcadas diferencias estacionales del 
proceso, se evalúo si existían diferencias significativas en la evapotranspiración promedio de los ecosistemas para las estaciones primavera, verano, otoño e invierno por separado. Se analizó la relación entre la diversidad funcional, evaluada por medio de los índices media comunitaria ponderada y divergencia funcional, y la evapotranspiración total anual de cada tipo de ecosistema, considerando sus tres repeticiones.

\section{ANÁLISIS ESTADÍSTICO}

Los resultados fueron evaluados mediante análisis de varianza para determinar si existían diferencias significativas entre comunidades, en términos de diversidad funcional y evapotranspiración (ET), con un nivel de significancia del 95 $\%$. Para el análisis se utilizó el software estadístico InfoStat. Se analizó en primera instancia la distribución de los datos mediante un test de Shapiro-Wilks, para verificar la normalidad de los datos. Se realizó posteriormente un análisis post-hoc, considerando test de Tukey, con el fin de comparar las medias entre las variables evaluadas. Considerando las marcadas diferencias estacionales del proceso, se evalúo si existían diferencias significativas también en la evapotranspiración promedio de los ecosistemas para las estaciones primavera, verano, otoño e invierno por separado. Finalmente, la relación entre ET e índices de diversidad funcional variables se determinó mediante correlación lineal de Pearson.

\section{RESULTADOS}

Los fragmentos de bosque nativo analizados presentaron diferencias en los rasgos fenológicos. Aun cuando los tres tipos de ecosistemas presentan fenología mixta siempreverde caducifolia, la proporción en que domina una u otra estrategia varía entre ecosistemas (Tabla 1).

\section{DiVERSIDAD FUNCIONAL}

Los índices de diversidad evaluados: media comunitaria ponderada (CWM) y divergencia funcional (FDvg), mostraron diferencias significativas (Fig. 2). Entre los tipos de ecosistemas analizados. Específicamente el Bosque primario dominado por Araucaria araucana (E1) presenta un CWM-SLA significativamente menor que los bosques dominados por Nothofagus dombeyi (ANDEVA una vía: $P=0,003$; Fig. 2a); sin embargo, este tipo de bosque presenta una divergencia del SLA (FDvg - SLA) significativamente mayor que el Bosque secundario mixto de N. dombeyi (ANDEVA una vía: $P<0.001$; Fig. 2b).

TABLA 1. Ecosistemas boscosos seleccionados para el estudio, composición del dosel superior, especies dominantes $\left({ }^{*}\right)$ y fenología. / Forest ecosystems selected for the study, composition of the canopy, dominants species $(*)$ and phenology.

\begin{tabular}{|c|c|c|c|c|}
\hline Ecosistema & Estructura bosque & Especies dominantes & Fenología & Proporción fenología \\
\hline \multirow{4}{*}{ E1 } & \multirow{4}{*}{ Bosque primario } & ${ }^{*}$ Araucaria araucana & Siempreverde & \multirow{4}{*}{$\begin{array}{l}75 \% \text { siempreverde } \\
25 \% \text { caducifolia }\end{array}$} \\
\hline & & Nothofagus dombeyi & Siempreverde & \\
\hline & & Nothofagus pumilio & Caducifolia & \\
\hline & & Lomatia hirsuta & Siempreverde & \\
\hline \multirow{5}{*}{ E2 } & \multirow{5}{*}{ Bosque primario } & *Nothofagus dombeyi & Siempreverde & \multirow{5}{*}{$\begin{array}{c}60 \% \text { siempreverde } \\
40 \% \text { caducifolia }\end{array}$} \\
\hline & & Nothofagus alpina & Caducifolia & \\
\hline & & Nothofagus obliqua & Caducifolia & \\
\hline & & Dasyphyllum diacanthoides & Siempreverde & \\
\hline & & Laureliopsis philippiana & Siempreverde & \\
\hline \multirow{4}{*}{ E3 } & \multirow{4}{*}{ Bosque secundario } & ${ }^{*}$ Nothofagus dombeyi & Siempreverde & \multirow{4}{*}{$\begin{array}{l}50 \% \text { siempreverde } \\
50 \% \text { caducifolia }\end{array}$} \\
\hline & & Persea lingue & Siempreverde & \\
\hline & & Nothofagus obliqua & Caducifolia & \\
\hline & & Nothofagus alpina & Caducifolia & \\
\hline
\end{tabular}




\section{EVAPOTRANSPIRACIÓN}

Los métodos utilizados para evaluar evapotranspiración (in situ y ex situ) presentaron una relación significativa $r^{2}=$ 0,98 y $P<0,00$ (Fig. 3). El análisis estacional del proceso de evapotranspiración arrojó diferencias significativas entre ecosistemas. En la Fig. 4a, correspondiente al total de ET promedio para el verano de 2014, se observan diferencias significativas entre el ecosistema E1 (ANOVA una vía: $P=$ 0,003; Fig. 4a) y los ecosistemas E2 y E3. En primavera de

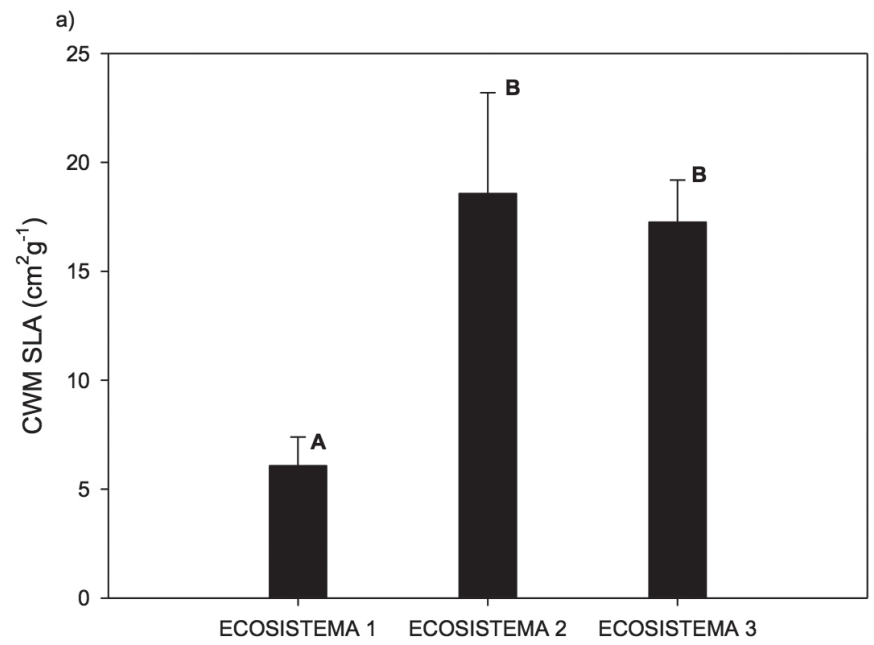

b)

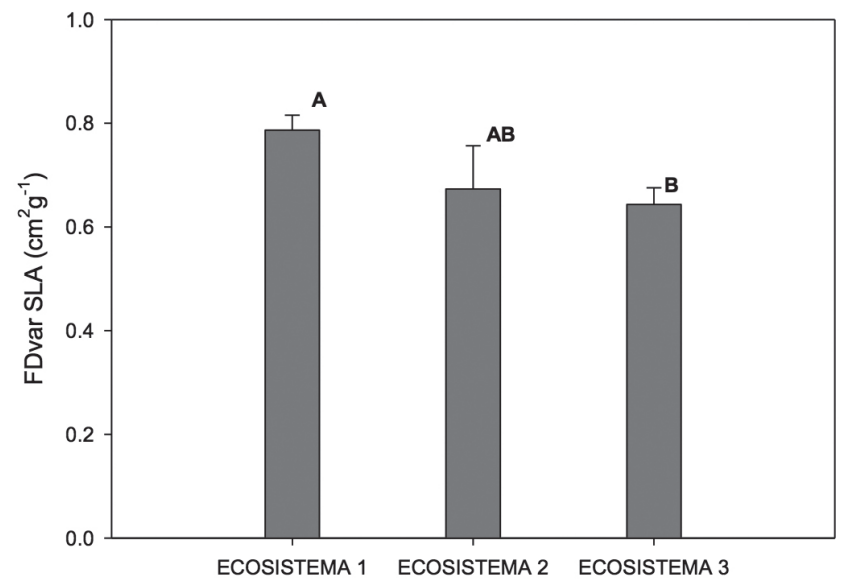

Figura 2. Valores promedio ( $\pm \mathrm{ES})$ de a) CWM-SLA $\left(\mathrm{cm}^{2} \mathrm{~g}^{-1}\right)$ y b) FDvg-SLA $\left(\mathrm{cm}^{2} \mathrm{~g}^{-1}\right)$ de los tres tipos forestales de bosque nativo (ver detalles en Tabla 1). Las comunidades que no presentan diferencia significativa en el índice de diversidad funcional comparten la misma letra $(P<0,01$; prueba Tukey HSD). / Average values ( \pm ES) of the a) CWM-SLA $\left(\mathrm{cm}^{2} \mathrm{~g}^{-1}\right)$ and b) FDvgSLA $\left(\mathrm{cm}^{2} \mathrm{~g}^{-1}\right)$ of the three forest types of native forest (see details in Table 1). Communities that do not present significant differences in the functional diversity index share the same letter $(P<0.01$; Tukey HSD test).
2014 (Fig. 4b) se observa que los bosques primarios E1 y E2 tienen similares valores de ET total, mientras los bosques secundarios E3 presentan diferencias significativas con un menor ET total (ANOVA una vía: $P=0,003$; Fig. $4 b$ ).

En el período de senescencia, otoño e invierno, los tres ecosistemas presentan similares valores de ET total, no presentando diferencias significativas, sin embargo, los bosques primarios mantienen ET total mayor que el bosque secundario. Cabe destacar que, en las cuatro estaciones del año, el ecosistema que presenta mayor ET total es el bosque primario dominado por Araucaria araucana.

\section{RELACIÓN ENTRE DIVERSIDAD FUNCIONAL Y EVAPOTRANSPIRACIÓN}

La relación entre la diversidad funcional, evaluada por medio de los índices CWM y FDvg, y la evapotranspiración total anual de cada tipo forestal, indica que ET y CWMSLA presentan una relación significativa inversa (Fig. 5), es decir que a mayor CWM-SLA menor es la ET total anual. Sin embargo, no se encontró una relación significativa entre la ET total anual y la FDvg. Considerando las diferencias en la proporción siempreverde-caducifolia de los distintos ecosistemas y que la hipótesis de relación de masa es la que mejor explica el proceso de evapotranspiración, se analizó la relación entre la evapotranspiración total y la proporción de ambas estrategias fenológicas en cada ecosistema (Fig. 6), la que presentó una relación positiva significativa.

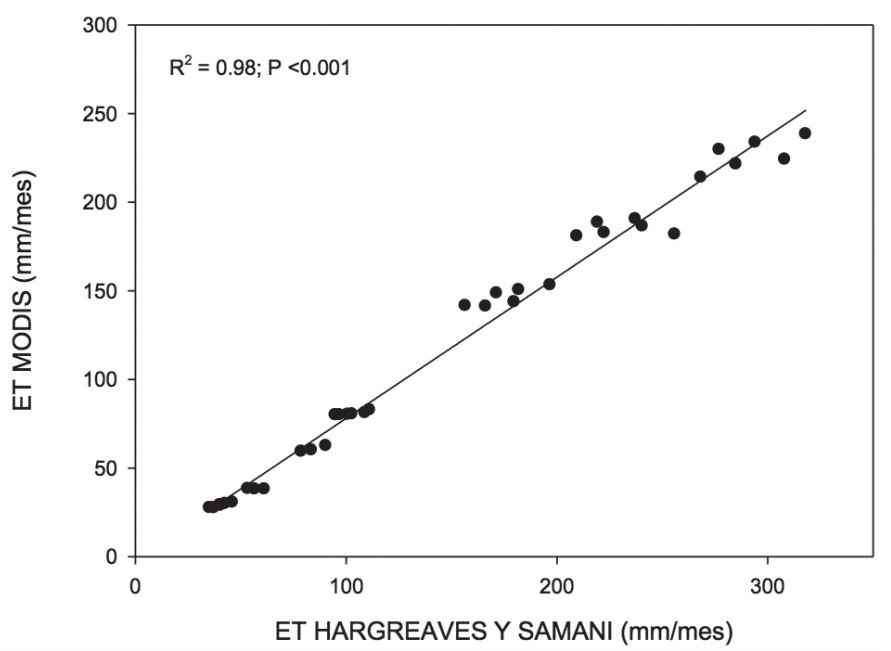

Figura 3. Ajuste entre evapotranspiración in situ (Hargreaves \& Samani 1985) y ex situ (MODIS). / Adjustment between evapotranspiration in situ (Hargreaves \& Samani 1985) and ex situ (MODIS). 

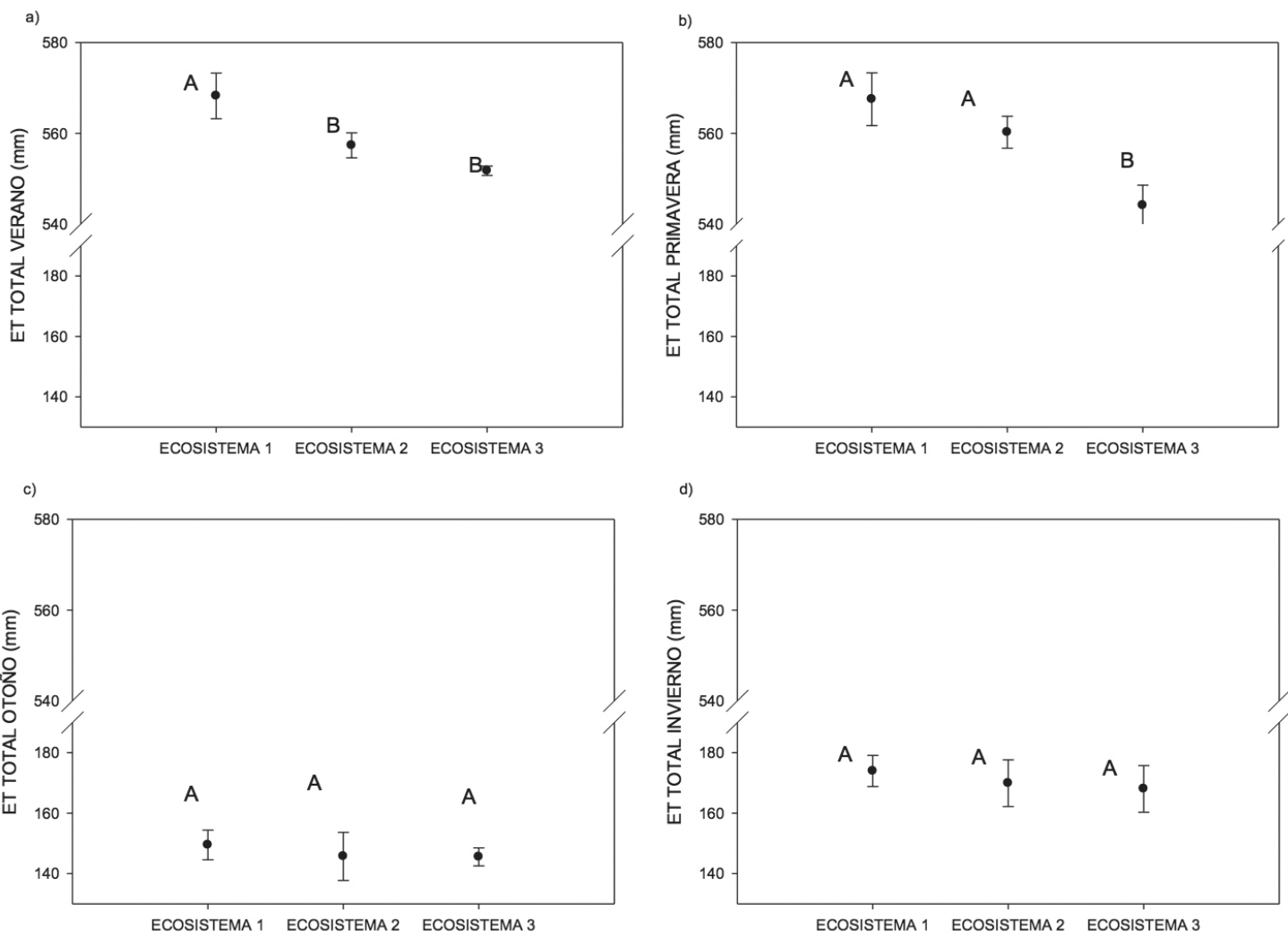

Figura 4. Valores promedio ( $\pm \mathrm{ES}$ ) de evapotranspiración del dosel (ET, mm) de los tres tipos de bosque nativo para las estaciones: a) verano, b) primavera, c) otoño, y d) invierno, año 2014. Las comunidades que no presentan diferencias significativas en la evapotranspiración comparten la misma letra $(P<0,01$; prueba Tukey HSD). / Average values ( \pm ES) of canopy evapotranspiration (ET, $\mathrm{mm}$ ) of the three types of native forest for the seasons: a) summer, b) spring, c) autumn, and d) winter, year 2014. Communities that do not present significant differences in evapotranspiration, they share the same letter $(P<0.01$, Tukey HSD test).

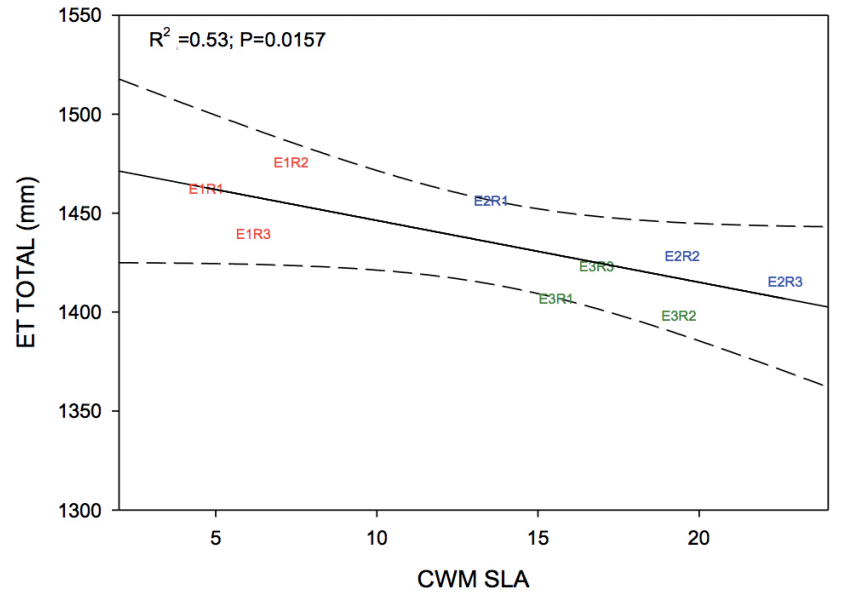

Figura 5. Relación entre ET total y CWM - SLA $\left(\mathrm{cm}^{2} \mathrm{~g}^{-1}\right)$. La relación se realizó agrupando las parcelas de cada repetición para tipos de bosque en función del CWM-SLA. Valores de $r^{2} y$ P corresponden a la correlación lineal de Pearson. / Relationship between total ET and CWM - SLA $\left(\mathrm{cm}^{2} \mathrm{~g}^{-1}\right)$. The relationship was made by grouping the parcels of each repetition for forest types according to the CWM-SLA. Values of $\mathrm{r}^{2}$ and $\mathrm{P}$ correspond to Pearson's linear correlation.

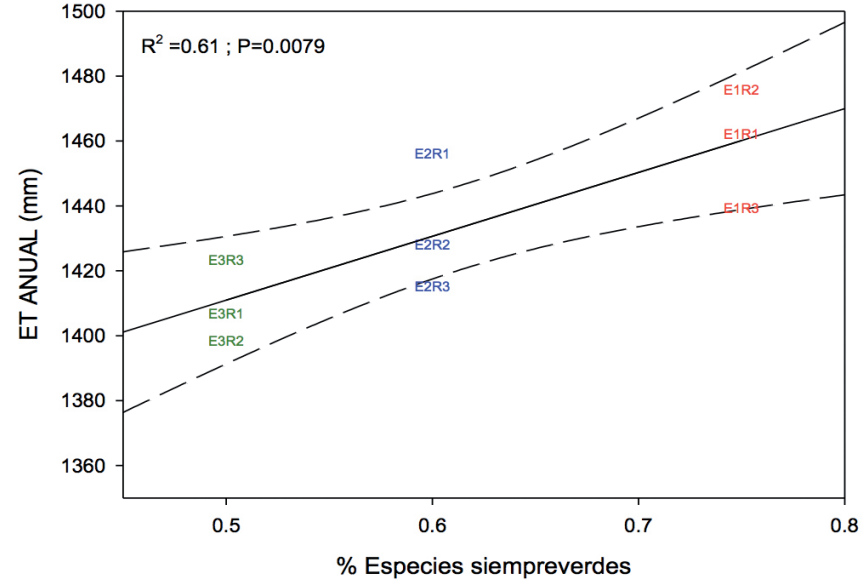

FiguRA 6. Relación entre el porcentaje de especies siempreverdes y la evapotranspiración anual total (mm). Valores de $r^{2}$ y $P$ corresponden a la correlación lineal de Pearson. / Relationship between the percentage of evergreen species and total annual evapotranspiration $(\mathrm{mm})$. Values of $\mathrm{r}^{2}$ and $\mathrm{P}$ correspond to Pearson's linear correlation. 


\section{DISCUSIÓN}

Los resultados muestran que las unidades boscosas, dependiendo de sus especies dominantes, son funcionalmente diferentes, y por ende, su evapotranspiración total y estacional es significativamente distinta. Además, existe una relación directa entre la diversidad funcional y la evapotranspiración.

El bosque primario dominado por Araucaria araucana (ecosistema 1) presentó mayor diversidad funcional, explicada en los índices divergencia funcional y media comunitaria ponderada, que los bosques primarios y secundarios dominados por Nothofagus dombeyi (ecosistemas 1 y 2), los que no presentan diferencias significativas entre sí. Sin embargo, se observó en estos últimos una mayor diversidad funcional en el bosque primario que en el bosque secundario. La principal diferencia en rasgos funcionales de los tres tipos de bosques corresponde a la representatividad de las estrategias fenológicas de las especies que dominan el dosel, ya que el ecosistema 1 presentó un $75 \%$ de especies siempreverde, ecosistema 2, 60 \% y ecosistema 3, $50 \%$. Las diferentes proporciones entre ambas estrategias influencia el proceso evapotranspiración, que depende en gran medida de los atributos foliares de las especies (Martín-López et al. 2007; Schulze et al. 2005). Un ecosistema con mayor presencia de especies siempreverde evapotranspirará más que uno con mayor presencia de caducifolias, debido a que tendrá mayor capacidad de intercepción y transpiración anual (Schulze et al. 2005).

De acuerdo con lo anterior, la variación en la evapotranspiración entre los tres ecosistemas evaluados se explica principalmente por los rasgos foliares de las especies dominantes y sus respectivas abundancias, así como también por la proporción de especies siempreverdes en el dosel. Queda manifiesto que la hipótesis de relación de masas (Grime 1998), evaluada mediante el índice media comunitaria ponderada del área foliar específica, tiene mayor incidencia en el proceso ecosistémico evapotranspiración que la hipótesis de diversidad (Tilman et al. 1997), evaluada mediante el índice de divergencia funcional del área foliar específica. Por lo tanto, es el rasgo funcional dominante y no la diferenciación de nicho ecológico el que determina la magnitud del proceso evapotranspiración. Estos resultados coinciden con lo establecido por Mokany et al. (2008), respecto a que son los rasgos funcionales de las especies dominantes y la contribución de dichas especies a la biomasa total de la comunidad, las que tendrían un mayor efecto sobre los procesos ecosistémicos.

Para este estudio, el proceso evapotranspiración tiene una relación inversa a la media comunitaria ponderada del área foliar específica, por lo que a menor valor de este rasgo, mayor es la tasa del proceso. Este resultado parece contra intuitivo ya que implica que el valor promedio del área foliar específica de la especie dominante más abundante, es comparativamente bajo. Un valor bajo de área foliar específica indica una menor inversión en área foliar por unidad de biomasa y una tasa de crecimiento relativo menor, y por ende una menor tasa fotosintética y menor transpiración asociada (Lambers et al. 2008). Sin embargo, estas características funcionales describen además la estrategia fenológica de las especies, ya que las especies caducifolias presentan valores de área foliar específica más altos que las siempreverdes (Dwyer et al. 2014). Especies siempreverdes mantienen el follaje, por lo que su capacidad de intercepción es mayor, lo que influye en las tasas de evaporación desde la superficie foliar. Por lo tanto, de acuerdo con los resultados, la dominancia de especies siempreverdes determina una mayor tasa de evapotranspiración. Esta relación explicaría la relación negativa entre la tasa de evapotranspiración y la media comunitaria ponderada, por lo que cuando el valor medio ponderado del área foliar específica de las especies dominantes en un tipo de bosque es bajo, como en el caso del ecosistema 1, dominado en un $75 \%$ por especies siempreverdes, presenta comparativamente mayor evapotranspiración que los otros ecosistemas con mayor proporción de caducifolias (Tabla 1). Por lo anterior, se infiere que, considerando que los bosques evaluados corresponden a ensambles de especies mixtas (caducifolio-siempreverde), es el grado en que predomina una u otra estrategia lo que influencia el proceso evapotranspiración.

Las diferentes tasas de evapotranspiración entre los bosques evaluados presentan además un patrón estacional. El ecosistema 1 presenta, comparativamente, mayor evapotranspiración que los tipos de bosques con mayor presencia de caducifolias en todas las estaciones del año. En las estaciones de crecimiento, primavera-verano, se observó una clara diferenciación en las tasas de evapotranspiración de los tres tipos de bosques. El proceso de evapotranspiración disminuye en otoño-invierno, lo que se explicaría en la menor actividad fisiológica en esta época. Aun cuando no existen diferencias significativas entre bosques en este periodo, el dosel dominado por mayor proporción de especies siempreverdes mantiene mayores tasas de evapotranspiración.

Respecto a la relación entre diversidad funcional y evapotranspiración, el ecosistema 1 presentó mayores tasas de evapotranspiración anual y estacional que los ecosistemas dos y tres. Esto indica que se cumple el segundo supuesto, ya que a mayor diversidad funcional se obtuvo mayor productividad ecosistémica. La diversidad funcional explica la magnitud del proceso no tan solo en el total anual, sino 
que también apoya la relación en el análisis estacional. En general, los bosques primarios presentaron mayores tasas de evapotranspiración que el bosque secundario.

Considerando que la diversidad funcional es el componente que mejor explica los efectos de la biodiversidad en los bienes y servicios ecosistémicos, sean éstos de regulación, provisión e incluso culturales y que se espera que a mayor diversidad funcional la productividad ecosistémica sea mayor (Díaz \& Cabido 2001; Hooper \& Vitousek 1997; Tilman et al. 1997), queda manifiesto que la capacidad de provisión de servicios de los bosques evaluados varía en función de las especies que componen el ecosistema.

Las marcadas diferencias funcionales de los bosques evaluados y su clara relación con el proceso ecosistémico evapotranspiración, sugieren que es necesario considerar la variación de la biodiversidad en estructura, composición y función, en la evaluación de procesos ecosistémicos, lo que permitirá conocer adecuadamente el funcionamiento ecosistémico y enfocar los esfuerzos de conservación y/o restauración en base a las características específicas de cada tipo de bosque.

La diversidad biológica se está perdiendo a un ritmo sin precedentes a nivel mundial debido a los cambios de uso del suelo y a la deforestación de los bosques, y hay evidencias de que la pérdida o disminución de algún componente de la biodiversidad puede tener diferentes efectos en el funcionamiento ecosistémico (Wright 2004). Muchos de los efectos son impredecibles y pueden significar una amenaza para la provisión de servicios, por lo anterior es importante tener en cuenta que los bosques nativos, aun cuando no hubiesen sido alterados, tienen respuestas ecosistémicas diferenciadas y que las especies que los componen y sus rasgos son los que marcan la diferencia.

\section{Conclusiones}

Los resultados de este estudio sugieren que la diversidad funcional, incluida en esta aproximación como la estrategia de fenología foliar de las especies dominantes, debe ser considerada cuando se evalúan los servicios ecosistémicos de regulación hídrica que provee el bosque nativo, ya que la provisión de estos está estrechamente vinculada con la biodiversidad. Por otra parte, la CWM-SLA de las comunidades forestales estudiadas explica de buena manera la diferenciación del proceso de evapotranspiración entre los ecosistemas. El rol de estos atributos funcionales adquiere mayor importancia si se considera que el bosque nativo sigue estando sometido a importantes presiones, y su degradación conlleva a cambios sustanciales en la función ecosistémica y la provisión de servicios. Finalmente, considerando que los rasgos de las plantas tienen el potencial para explicar la variación en procesos de intercambio ecosistema-atmósfera, nuestro estudio contribuye a comprender cuál es el papel de las propiedades funcionales del bosque nativo, a partir del estudio de algunos tipos forestales como modelo para describir las implicancias en su funcionamiento. Futuros estudios de este tipo debieran ampliar el número de tipos forestales evaluados, considerar un periodo más largo que un año en la variación estacional de la evapotranspiración inferida con el uso de sensores remotos, y considerar el rol de las especies del sotobosque y epífitas en los flujos de materia y energía del bosque. Finalmente, es importante destacar que este tipo de estudios destacan la importancia que la relación entre biodiversidad y funcionamiento ecosistémico puede tener en la conservación del bosque nativo, ya que dicha relación es una excelente razón para conservar biodiversidad local a nivel local, regional y global.

\section{AGRADECIMIENTOS}

Este estudio se realizó en el marco del proyecto Fondecyt 1140455 y tuvo apoyo metodológico del proyecto Fondecyt 1140531. El estudio fue parcialmente financiado por el Centro Internacional de Investigaciones para el Desarrollo (IDRC), en el marco del proyecto: "Evaluación del bienestar y los impactos económicos del cambio climático en el suministro y demanda de agua en Chile, Colombia y Bolivia". AP fue financiado parcialmente por CONICYT PFB-23 e ICM P05-002.

\section{REFERENCIAS}

Allen, R.G., Pereira, L.S., Raes, D., Smith, M. 1998. Crop Evapotranspiration-Guidelines for Computing Crop Water Requirements-FAO Irrigation and Drainage Paper 56. FAO, Rome, 300(9): D05109.

Baudena, M., Hardenberg J.V., Provenzale, A. 2013. Vegetation patterns and soil-atmosphere water fluxes in drylands. Advances in Water Resources 53: 131-138.

Butterfield, B.J., Suding, K.N. 2013. Single-trait functional indices outperform multi-trait indices in linking environmental gradients and ecosystem services in a complex landscape. Journal of Ecology 101: 9-17.

Cadotte, M.W., Carscadden, K., Mirotchnick, N. 2011. Beyond species: functional diversity and the maintenance of ecological processes and services. Journal of Vegetation Science 48: 1079-1087.

Casanoves F., Pla L., Di Rienzo, J.A., Díaz, S. 2011. FDiversity: a software package for the integrated analysis of functional 
diversity. Methods in Ecology and Evolution 2: 233-237.

Chávez, P.S. 1996. Image-Based Atmospheric Corrections Revisited and Improved. Photogrammetric Engineering and Remote Sensing. 62. 1025-1036.

Díaz, S., Cabido, M. 2001. Vive la difference: plant functional diversity matters to ecosystem processes. Trends in Ecology \& Evolution 16: 646-655.

Díaz, S., Fargione, J., Chapin, F.S., Tilman, D. 2006. Biodiversity loss threatens human well-being. PLoS Biology 4(8): e277.

Díaz, M.F., Bigelow, S.W., Armesto, J.J. 2007. Alteration of the hydrologic cycle due to forest clearing and its consequences for rainforest succession. Forest Ecology and Management 244: 32-40.

Dwyer, J.M., Hobbs, R.J., Mayfield, M.M. 2014. Specific leaf area responses to environmental gradients through space and time. Ecology 95(2): 399-410.

Fortunel, C., Garnier, E., Joffre, R., Kazakou, E., Quested, H., Grigulis, K., Lavorel, S., Ansquer, P., Castro, H., Cruz, P., DoleŽal, J., Eriksson, O., Freitas, H., Golodets, C., Jouany, C., Kigel, J., Kleyer, M., Lehsten, V., Lepš, J., Meier, T., Pakeman, R., Papadimitriou, M., Papanastasis, V.P., Quétier, F., Robson, M., Sternberg, M., Theau, J., Thébault, A. and Zarovali, M. 2009. Leaf traits capture the effects of land use changes and climate on litter decomposability of grasslands across Europe. Ecology 90: 598-611. doi:10.1890/08-0418.1

Garnier, E., Cortez, J., Billès, G., Navas, M.-L., Roumet, C., Debussche, M., Laurent, G., Blanchard, A., Aubry, D., Bellmann, A., Neill, C., Toussaint, J.P. 2004. Plant functional markers capture ecosystem properties during secondary succession. Ecology 85: 2630-2637.

Grime, J.P. 1998. Benefits of plant diversity to ecosystems: immediate, filter and founder effects. Journal of Ecology 86: 902-910.

Hantson, S., Chuvieco, E., 2011. Evaluation of different topographic correction methods for Landsat imagery. International Journal of Applied Earth Observation and Geoinformation 13: 691-700. https://doi.org/10.1016/j. jag.2011.05.001

Hargreaves, G.H. and Samani, Z.A. 1985. Reference Crop Evapotranspiration from Temperature. Applied Engineering in Agriculture, 1, 96-99. http://dx.doi. org/10.13031/2013.26773

Hein, L., Van Koppen K., De Groot, R.S., Van lerland, E.C. 2006. Spatial scales, stakeholders, and the valuation of ecosystem services. Ecological Economics 57: 209-228.

Hillebrand, H., Matthiessen, B. 2009. Biodiversity in a complex world: consolidation and progress in functional biodiversity research. Ecology Letters 12: 1405-1419.
Hooper, D.U., Vitousek, P.M. 1997. The effects of plant composition and diversity on ecosystem processes. Science 277: 1302-1305.

Hooper, D.U., Chapin, F.S., Ewell, J.J., Hector, A., Inchausti, P., Lavorel, S., Lawton, J.H., Lodge, D.M., Loreau, M., Naeem, S., Schmid, B., Setälä, H., Symstad, A.J., Vandermeer, J., Wardle, D.A. et al. 2005. Effects of biodiversity on ecosystem functioning: a consensus of current knowledge. Ecological Monographs 75: 3-35.

Jensen M.E., Burman, R.D., Allen, R.G., (Eds.) 1990: Evapotranspiration and irrigation water requirements. ASCE Manuals and Reports on Engineering Practice 70.

Kelliher, F.M., Leuning, R., Raupach, M.R., Schulze, E.D. 1995. Maximum conductances for evaporation from global vegetation types. Agricultural and Forest Meteorology 73: 1-16.

Lambers, H., Chapin, F.S., Pons, T.L. 2008. Plant Physiological Ecology, Ed 2. Springer, New York. p 163-223.

Lara, A., Little, C., Urrutia, R., McPhee, J., Alvarez-Garreton, C., Oyarzún, C., Soto, D., Donoso, P., Nahuelhual, L., Pino, M., Arismendi, I. 2009. Assessment of ecosystem services as an opportunity for the conservation and management of native forests in Chile. Forest Ecology and Management 258(4): 415-424.

Lepš, J., De Bello, F., Lavorel, S., Berman, S. 2006. Quantifying and interpreting functional diversity of natural communities: practical considerations matter. Preslia 78: 481-501.

Luebert, F., Pliscoff, P. 2006. Sinopsis bioclimática y vegetacional de Chile. Editorial Universitaria, Santiago. 316 pp.

Martín-López, B., González, J.A., Díaz, S., Castro, I., GarcíaLlorente, M. 2007. Biodiversidad y bienestar humano: el papel de la diversidad funcional. Ecosistemas 16(3): 69-80.

Mason, N.W.H., Mouillot, D., Lee, W.G., Wilson, J.B. 2005. Functional richness, functional evenness and functional divergence: the primary components of functional diversity. Oikos 111: 112-118.

Mo, X., Liu, S., Chen, X., Hu, S. 2018. Variability, tendencies, and climate controls of terrestrial evapotranspirationand gross primary productivity in the recent decade over China. Ecohydrology 11: e1951. https://doi.org/10.1002/ eco.1951

Mokany, K., Ash, J., Roxburgh, S. 2008. Functional identity is more important than diversity in influencing ecosystem processes in a temperate native grassland. Journal of Ecology 96: 884-893.

Monteith, J.L. 1965. Evaporation and Environment. In: Fogg, B.D. (ed.). The State and Movement of Water in Living Organism, Symposium of the Society of Experimental biology. Vol. 19: 205-234. Cambridge University Press, Cambridge. UK. 
Pérez-Harguindeguy, N., Díaz, S., Garnier, E., Lavorel, S., Poorter, H., Jaureguiberry, P., Cornelissen, J.H.C. 2013. New handbook for standardized measurement of plant functional traits worldwide. Australian Journal of Botany 61(3): 167-234.

Petchey, O.L., Gaston, K.J. 2006. Functional diversity: back to basics and looking forward. Ecology Letters 9: 741-758.

Pieruschka, R., Huber, G., Berry, J.A. 2010. Control of transpiration by radiation. Proceedings of the National Academy of Sciences of the United States of America 107(30): 1337213377. doi:10.1073/pnas.0913177107

Poorter, H., Garnier, E. 1999. Ecological significance of inherent variation in relative growth rate. In: Pugnaire, F., Valladares, X. (eds.), Handbook of functional plant ecology. 81-120 pp. Marcel Dekker, New York, USA.

Reese, H., Olsson, H. 2011. C-correction of optical satellite data over alpine vegetation areas: A comparison of sampling strategies for determining the empirical c-parameter. Remote Sensing of Environment 115: 1387-1400. https:// doi.org/10.1016/J.RSE.2011.01.019

Reich, P.B., Walters, M.B., Ellsworth, D.S. 1997. From tropics to tundra: global convergence in plant functioning. Proceedings of the National Academy of Sciences of the United States of America 94: 13730-13734.

Reich, P.B., Wright, I.J., Cavender-Bares, J., Craine, J.M., Oleksyn, J., Westoby, M., Walters, M.B. 2003. The evolution of plant functional variation: traits, spectra, and strategies. International Journal of Plant Sciences 164(S3): 143-164.

Reichstein, M., Bahn, M., Mahecha, M.D., Kattge, J., Baldocchi, D.D. 2014. Linking plant and ecosystem functional biogeography. Proceedings of the National Academy of Sciences USA 111(38): 13697-13702.

Segura M, R., Trincado, G., 2003. Cartografía digital de la Reserva Nacional Valdivia a partir de imágenes satelitales Landsat TM. Bosque (Valdivia) 24, 43-52. https://doi. org/10.4067/S0717-92002003000200005

Stehr, A., Aguayo, M., Link, O., Parra, O., Romero, F., Alcayaga, H.
2010. Modelling the hydrologic response of a mesoscale Andean watershed to changes in land use patterns for environmental planning, Hydrological and Earth System Sciences 14: 1963-1977.

Schulze, E.D., Beck, E., Müller-Hohenstein, K. 2005. Plant Ecology. Springer Verlag, Heidelberg. 702 pp.

Teillet, P.M., Guindon, B., Goodenough, D.G. 1982. On the Slope-Aspect Correction of Multispectral Scanner Data. Canadian Journal of Remote Sensing 8: 84-106. https:// doi.org/10.1080/07038992.1982.10855028

Tilman, D. 2001. Functional diversity. In: Levin, S.A. (ed.). Encyclopedia of Biodiversity. Vol. 3: 109-120, Academic Press. Princeton University, New Jersey, U.S.A.

Tilman, D., Knops, J., Wedin, D., Reich, P., Ritchie, M., Siemann, E. 1997. The influence of functional diversity and composition on ecosystem processes. Science 277(5330): 1300-1302.

Tilman, D., Lehman, C. 2002. Biodiversity, Composition, and Ecosystem Processes: Theory and Concepts, p: 9-41. In: Kinzig, A.P., Pacala, S.W., Tilman, D (eds.), The functional consequences of biodiversity: Empirical progress and theoretical extensions. Princeton University Press, Princeton, NJ.

Violle, C., Navas, M.-L., Vile, D., Kazakou, E., Fortunel, C., Hummel, I., Garnier, E. 2007, Let the concept of trait be functional. Oikos 116(5): 882-892.

Ward, A.D., Trimble, S.W. 2003. Environmental Hydrology. Lewis Publishers. Second edition. 475 pp. Boca Raton, Florida. p. 83-117.

Wright, I. J., Reich, P. B., Westoby, M., Ackerly, David D., Baruch, Z., Bongers, F., Cavender-Bares, Jeannine, Chapin, F. S., Cornelissen, J. H. C., Diemer, M., Flexas, J., Garnier, E., Groom, P. K., Gulias, J., Hikosaka, K., Lamont, B. B., Lee, T., Lusk, C., Midgley, J. J., Navas, M. L., Niinemets, U., Oleksyn, J., Osada, N., Poorter, H., Poot, P. et al. 2004. World-wide leaf economics spectrum. Nature. 428:821827.

Received: 12.07 .2018

Accepted: 08.04.2019 